\title{
Evolución en el diagnóstico y manejo del esófago de Barrett: 2004-2014
}

\section{Evolution of the Diagnosis and Management of Barrett's Esophagus: 2004-2014}

Rodrigo Castaño Llano, MD. ${ }^{1}$

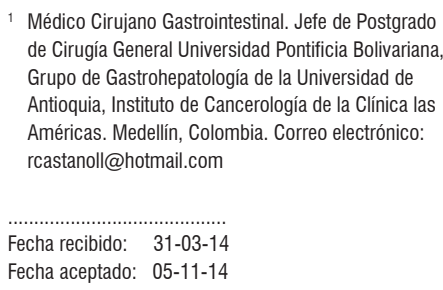

1 Médico Cirujano Gastrointestinal. Jefe de Postgrado de Cirugía General Universidad Pontificia Bolivariana Grupo de Gastrohepatología de la Universidad de Antioquia, Instituto de Cancerología de la Clínica las Américas. Medellín, Colombia. Correo electrónico: rcastanoll@hotmail.com

Fecha recibido: $\quad 31-03-14$ Fecha aceptado: $05-11-14$

El esófago de Barrett (EB) se diagnostica endoscópicamente cuando el epitelio escamoso lesionado crónicamente por el ácido es transdiferenciado ${ }^{1}$ a mucosa columnar (1). El epitelio metaplásico columnar, a menudo pero no siempre, semeja la metaplasia intestinal con células caliciformes como marcador histológico clave. La metaplasia intestinal en el esófago no debe confundirse con la metaplasia del cardias, la que no es infrecuente.

El diagnóstico del EB es esencialmente un diagnóstico endoscópico. Para un diagnóstico seguro del EB es importante identificar y localizar adecuadamente la unión esofagogástrica (UEG). Existen tres marcas anatómicas relevantes que ayudan a la identificación de la UEG:

1. La terminación proximal de los pliegues gástricos en el cardias.

2. Un ligero estrechamiento del esófago distal, a la altura del esfínter interno, conocido como el pinzamiento o la impronta esofágica.

3. La terminación distal de la vasculatura en empalizada en el esófago distal.

En una situación normal la UEG y la unión mucosa escamocolumnar se encuentran en la mitad entre el complejo esfinteriano o zona de alta presión, hecho de músculo liso del esfínter esofágico inferior y el músculo estriado de la crura diafragmática.

Normalmente, un esfínter está por encima del otro, en el caso de la hernia hiatal el esfínter interno se desplaza proximalmente respecto al esfínter externo (crura diafragmática). Una leve separación de ambos esfínteres solo puede detectarse por la manometría de alta resolución. La hernia hiatal se identifica por endoscopia cuando el esfínter interno es demostrable por encima de la impronta de la crura diafragmática en el hiato esofágico. Infortunadamente el diagnóstico endoscópico de la hernia hiatal está propenso al error por la sobreinsuflación, las arcadas o el vómito o la confusión en la vasculatura del cardias (frecuentemente no visible) con la vasculatura en empalizada del esófago distal. Las recomendaciones para un apropiado diagnóstico de hernia hiatal son:

1. Evaluar la unión GE durante una respiración tranquila

2. En ausencia de vómito o arcadas

3. Evitar la sobreinsuflación

4. Durante el retiro del endoscopio sin angulaciones

\footnotetext{
${ }^{1}$ La transdiferenciación tiene lugar cuando una célula que no es una célula madre se transforma a otro tipo de célula diferente o cuando una célula madre ya diferenciada crea células fuera de su ruta de diferenciación ya establecida; es un tipo de metaplasia que incluye todos los cambios en el destino de una célula.
} 
5. Visualizar y medir la distancia entre el esfínter interno (unión gastroesofágica) de la impresión de la crura diafragmática (esfínter externo).

Se espera que la adopción de estas recomendaciones disminuya el número de diagnósticos erróneos de hernia hiatal.

Cuando la UGE es claramente determinada, la mucosa columnar por encima de este nivel debe ser rotulada como metaplasia columnar o EB. La extensión de los cambios metaplásicos debe ser medida conforme los criterios de Praga, con el valor de $\mathrm{C}$ que corresponde a la extensión de la afección circunferencial y $\mathrm{M}$ por la máxima extensión del compromiso. Cuando los valores de $\mathrm{C}$ y $\mathrm{M}$ pueden establecerse con seguridad, términos como EB largo, corto o ultracorto no son necesarios. La documentación de la metaplasia intestinal con células caliciformes, como se requiere en las guías americanas para el diagnóstico de $\mathrm{BE}$, no es absolutamente necesaria por diversas razones (2), especialmente en el segmento corto de metaplasia, o por el desarrollo de cambios neoplásicos sin cambios de metaplasia intestinal adyacente (3).

La importancia de reconocer el EB radica en su condición precancerosa aunque el riesgo de desarrollar cáncer es actualmente menor a lo planteado en estudios previos (4). Este bajo riesgo actual en buena medida se explica por el amplio uso de los bloqueadores de bomba (5). Parece que la neoplasia se desarrolla desde la mucosa no displásica, vía displasia de bajo y alto grado hasta carcinoma invasivo. La displasia de bajo grado es sobrediagnosticada en la práctica diaria, debido a la confusión de los cambios inflamatorios y reactivos que pueden sugerir neoplasia. Las biopsias para descartar neoplasia deben obtenerse después de una terapia sostenida con supresores ácidos para disminuir la inflamación inducida por el RGE e idealmente deben ser evaluadas por patólogos expertos en tracto gastrointestinal. Preferiblemente, endoscopios de alta resolución deben ser usados para evaluar la mucosa. El uso mandatorio de las técnicas tipo NBI o afines es controvertido, aunque estas técnicas son las utilizadas en las unidades de referencia (6).

Los cambios neoplásicos pueden presentarse como una alteración focal visible de mucosa que debe graduarse de acuerdo con la clasificación de Paris. Las neoplasias tempranas más frecuentemente encontradas están ubicadas espacialmente entre las 2 y 5 del reloj (7). No es infrecuente que la neoplasia no sea detectable endoscópicamente. Las biopsias aleatorias de cuatro cuadrantes, cada uno a dos $\mathrm{cm}$ en el segmento de EB, se recomiendan para detectar cambios neoplásicos ocultos. Cuando una lesión es detectada, la estimación de la profundidad de la invasión por ultrasonido endoscópico no necesariamente debe determinarse para definir la intervención endoscópica (8).
Cualquier anomalía focal debe ser resecada endoscópicamente usando la técnica de bandas o disección endoscópica. Una evaluación experta del espécimen resecado y bien orientado permite una adecuada estimación de la profundidad de la infiltración en caso de crecimiento tumoral invasivo. La mucosa normal adyacente a la lesión debe ser completamente erradicada con radiofrecuencia circunferencial o parcial. La supresión ácida debe continuarse para permitir la cicatrización de la mucosa escamosa y puede prevenir la recurrencia de la metaplasia columnar. Los resultados a largo plazo parecen ser gratificantes $(9,10)$.

Debe tenerse en cuenta antes de la RFA del EB con displasia de alto o bajo grado, diferentes escenarios que redundarán en unos mejores resultados:

1. Solo los pacientes con displasia de bajo grado bien documentada y con la concordancia de dos patólogos deben ser intervenidos. De los pacientes con diagnóstico inicial de DBG, solo $15 \%$ tienen la confirmación en centros con patólogos expertos (11-13) además, no obstante la confirmación de la DBG por patólogos expertos un gran porcentaje (28\%) muestra regresión en el tiempo, como lo sugiere Phoa (14), en este estudio la confirmación histológica en solo una ocasión fue el más importante criterio de selección para la terapia. Sin embargo, la selección de pacientes con DBG en varias endoscopias refinaría el proceso de seleccionar pacientes con displasia y con riesgo de progresión. Además, la progresión de la DBG a DAG hace de la RFA un procedimiento que vale la pena tener en cuenta.

2. Se necesitan mejores métodos para discriminar el riesgo de progresión en los pacientes con EB con o sin displasia. El análisis multivariado en otro artículo de Phoa (15) determina que el EB circunferencial, tiempo con el diagnóstico de EB, el tiempo con el diagnóstico de la displasia son predictores de progresión. Sin embargo, marcadores, biológicos, moleculares e histológicos para identificar los candidatos a las terapias ablativas y/o de resección son una prioridad en la clínica e investigación (16-18).

3. La radiofrecuencia no elimina totalmente el riesgo de persistencia o progresión del EB; una cuarta parte de los pacientes en diferentes publicaciones requieren de intervenciones endoscópicas adicionales para completar la eliminación del tejido (19). Pero estas reintervenciones no disminuyen el valor de la RFA, sino que reflejan el carácter único y complejo del comportamiento anatómico y biológico del EB.

4. El seguimiento en estos pacientes está marcado por la posibilidad de recurrencia y no hay un protocolo de seguimiento ni en la técnica endoscópica ni el tiempo que sea bien establecido para estos pacientes (20). Tanto los pacientes como los médicos deben tener 
un alto compromiso al momento de embarcarse en un manejo proactivo del EB con displasia. Un mayor número de intervenciones se relaciona con una menor calidad de vida por los efectos adversos de la ablación y la resección; sin embargo, hay implicaciones psicológicas intrínsecas en los pacientes que viven con una enfermedad con una alta tasa de progresión.

5. En la actualidad, ninguno de los estudios de RFA en el EB displásico no evalúan el mayor impacto clínico de la terapia como es la aparición de cáncer o la muerte por cáncer, aunque la valoración de este tópico tiene implicaciones éticas cuestionables. El cáncer de esófago cursa con metástasis tempranas, alta morbimortalidad perioperatoria y una tasa de sobrevida menor a $20 \%$ a 5 años.

6. Las publicaciones casi exclusivas de centros expertos en estas terapias hacen los resultados menos reproducibles a la población general en la práctica diaria.

En el presente número se muestra la evolución en el enfoque y manejo de los pacientes con EB asociado a displasia o el carcinoma in situ; inicialmente la terapia se centró en la ablación con argón plasma con resultados poco alentadores por la alta recurrencia asociada a los EB más largos. Se implementó la práctica de la mucosectomía con bandas y se complementaba con argón plasma con resultados más alentadores y en la actualidad la terapia se hace en el compromiso circunferencial con RFA y en los casos de nodulaciones con la mucosectomía con bandas seguida por RFA. Los diferentes estudios proveen importante evidencia para apoyar el uso de la ablación por radiofrecuencia no solo en pacientes con displasia de alto grado y el cáncer temprano, también para la displasia de bajo grado confirmada y en los pacientes seleccionados con EB. Un enfoque proactivo endoscópico para eliminar la displasia puede resultar en la reducción de la morbilidad y la mortalidad relacionadas con la progresión de esta enfermedad.

\section{REFERENCIAS}

1. Clemons NJ, Koh SY, Phillips WA. Advances in understanding the pathogenesis of Barrett's esophagus. Discovery medicine 2014; 17: 7-14.

2. Spechler SJ. Barrett's esophagus: is the goblet half empty? Clinical gastroenterology and hepatology: the official clinical practice journal of the American Gastroenterological Association 2012; 10: 1237-8.

3. Westerhoff M, Hovan L, Lee C, Hart J. Effects of dropping the requirement for goblet cells from the diagnosis of Barrett's esophagus. Clinical gastroenterology and hepatology: the official clinical practice journal of the American Gastroenterological Association 2012; 10: 1232-6.

4. Hvid-Jensen F, Pedersen L, Drewes AM, Sorensen HT, Funch-Jensen P. Incidence of adenocarcinoma among patients with Barrett's esophagus. The New England journal of medicine 2011; 365: 1375-83.

5. Kastelein F, Spaander MC, Steyerberg EW, et al. Proton pump inhibitors reduce the risk of neoplastic progression in patients with Barrett's esophagus. Clinical gastroenterology and hepatology: the official clinical practice journal of the American Gastroenterological Association 2013; 11: 382-8.

6. Boerwinkel DF, Swager AF, Curvers WL, Bergman JJ. The Clinical Consequences of Advanced Imaging Techniques in Barrett's Esophagus. Gastroenterology 2014.

7. Kariyawasam VC, Bourke MJ, Hourigan LF, et al. Circumferential location predicts the risk of high-grade dysplasia and early adenocarcinoma in short-segment Barrett's esophagus. Gastrointestinal endoscopy 2012; 75: 938-44.

8. Bergeron EJ, Lin J, Chang AC, Orringer MB, Reddy RM. Endoscopic ultrasound is inadequate to determine which $\mathrm{T} 1 / \mathrm{T} 2$ esophageal tumors are candidates for endoluminal therapies. The Journal of thoracic and cardiovascular surgery 2014; 147: 765-71: Discussion 71-3.

9. Orman ES, Li N, Shaheen NJ. Efficacy and durability of radiofrequency ablation for Barrett's Esophagus: systematic review and meta-analysis. Clinical gastroenterology and hepatology: the official clinical practice journal of the American Gastroenterological Association 2013; 11: 1245-55.

10. Orman ES, Kim HP, Bulsiewicz WJ, et al. Intestinal metaplasia recurs infrequently in patients successfully treated for Barrett's esophagus with radiofrequency ablation. The American journal of gastroenterology 2013; 108: 187-95; quiz 96.

11. Downs-Kelly E, Mendelin JE, Bennett AE, et al. Poor interobserver agreement in the distinction of high-grade dysplasia and adenocarcinoma in pretreatment Barrett's esophagus biopsies. The American journal of gastroenterology 2008; 103: 2333-40; quiz 41.

12. Sikkema M, Looman CW, Steyerberg EW, et al. Predictors for neoplastic progression in patients with Barrett's Esophagus: a prospective cohort study. The American journal of gastroenterology 2011; 106: 1231-8.

13. Curvers WL, ten Kate FJ, Krishnadath KK, et al. Low-grade dysplasia in Barrett's esophagus: overdiagnosed and underestimated. The American journal of gastroenterology 2010; 105: 1523-30.

14. Phoa KN, Pouw RE, van Vilsteren FG, et al. Remission of Barrett's esophagus with early neoplasia 5 years after radiofrequency ablation with endoscopic resection: a Netherlands cohort study. Gastroenterology 2013; 145: 96-104.

15. Phoa KN, van Vilsteren FG, Weusten BL, et al. Radiofrequency ablation vs endoscopic surveillance for patients with Barrett esophagus and low-grade dysplasia: a randomized clinical trial. JAMA: the journal of the American Medical Association 2014; 311: 1209-17.

16. Mokrowiecka A, Wierzchniewska-Lawska A, Smolarz B, et al. Amplification of Her-2/neu oncogene in GERD Barrett's metaplasia - dysplasia - adenocarcinoma sequence. Hepato-gastroenterology 2013; 60: 1063-6.

17. Bird-Lieberman EL, Dunn JM, Coleman HG, et al. Population-based study reveals new risk-stratification bio- 
marker panel for Barrett's esophagus. Gastroenterology 2012; 143: 927-35 e3.

18. Prasad GA, Wang KK, Halling KC, et al. Utility of biomarkers in prediction of response to ablative therapy in Barrett's esophagus. Gastroenterology 2008; 135: 370-9.

19. Lee JK, Cameron RG, Binmoeller KF, et al. Recurrence of subsquamous dysplasia and carcinoma after successful endoscopic and radiofrequency ablation therapy for dysplastic Barrett's esophagus. Endoscopy 2013; 45: 571-4.

20. Álvarez Herrero L, Curvers WL, Bisschops R, et al. Narrow band imaging does not reliably predict residual intestinal metaplasia after radiofrequency ablation at the neo-squamo columnar junction. Endoscopy 2014; 46: 98-104. 